\title{
ORIENTIERUNG
}

\section{Fachlichkeit und Effizienz}

\author{
Jörg Meese und Wolfgang Nitze
}

Aus Sorge vor einer Entwertung Sozialer Arbeit haben die beiden Autoren Jörg Meese und Wolfgang Nitze zahlreiche Führungskräfte nach ihren "Ansichten zur Lage im Psychosozialen System " befragt und die Ergebnisse in dem Buch »Mutmacher zusammengestellt.

Der »Mutmacher « ist kein Buch ausschließlich für Führungskräfte, sondern die Beiträge sollen alle Menschen ansprechen, die sich im psychosozialen Bereich bewegen. Der Sammelband enthält aber sicher Anregungen, die besonders für Führungskräfte wertvoll sein können. Dieser Beitrag, aufbauend auf den Erfahrungen mit dem Buch »Mutmacher «, kann Einblicke geben, wie Führungskräfte sozialer Organisationen ihre Situation einschätzen und welche persönlichen Eigenschaften sie für ihre Arbeit für besonders wichtig erachten.

Der »Mutmacher « sucht unter anderem nach einer Antwort auf die Frage, ob Effektivität und partnerschaftliches Handeln unter Berücksichtigung ethischer und moralischer Erfordernisse in sozialen Dienstleistungsunternehmen eine gemeinsame Basis haben und ob dies ein Fundament für eine Neuorientierung psychosozialer Arbeit mit sich bringen kann. Unsere Erfahrungen aus der praktischen Arbeit zeigen, dass wir mit der bisherigen politischen, sozialpädagogischen, medizinischen oder therapeutischen Arbeit in der Praxis an Grenzen stoßen, die uns daran hindern, das zu tun, wofür wir ausgebildet wurden und was für uns im Arbeitsalltag von Bedeutung ist.

Es entsteht der Eindruck, dass unter der momentanen Entwicklung in der psychosozialen Arbeit wesentliche Attribute wie Spontaneität, Kreativität und damit die Qualität und unser Engagement zu leiden begonnen haben. Durch Rückgriff auf Erfahrungen im Rahmen unserer Tätigkeit in einem Berliner Bezirk soll unser Ansatz verdeutlicht werden. Bisher hatten wir Ziele und Entwicklungsmöglichkeiten in den jeweiligen Gremien unter Beteiligung der fachlichen und politischen Entscheidungsträger gemeinsam definiert. Grundannahmen hinsichtlich der Hilfen für behinderte Menschen und die Weiterentwicklung von Angebotsstrukturen konnten beraten und veröffentlicht werden. Problembewusstsein wurde in Fortbildungen geschärft; Beraterinnen und Berater konnten gewonnen werden. Betroffene wurden ermuntert, sich frühzeitig in Entwicklungsprozesse einzubringen, um Teilhabe so früh wie möglich zu gewährleisten. Probleme in den Einrichtungen und im Bezirk wurden systematisch auf den entsprechenden Ebenen thematisiert.

So wurden bedeutsame Vorgehensweisen über Jahre entwickelt, gepflegt, umgesetzt: systemumfassendes Den-

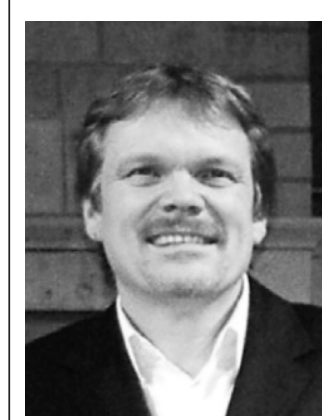

Jörg Meese (44) hat Sozialarbeit an der Alice-SalomonFachhochschule in Berlin studiert und berufsbegleitende Ausbildungen zum Mediator (IKOM) sowie zum EOQ-Qualitätsauditor TÜV absolviert. Er war bei der Stiftung SPI in Berlin als Koordinator bei der Clearingstelle Jugendhilfe und Polizei sowie als Leiter des Betreuten Einzelwohnen für chronisch mehrfachbeeinträchtigte abhängigkeitskranke Menschen in Berlin-Friedrichshain tätig. Seit zwei Jahren leitet er die Stabsstelle Qualitätsmanagement beim Berliner Landesverband der Arbeiterwohlfahrt. E-Mailmeese.joerg@gmx.de

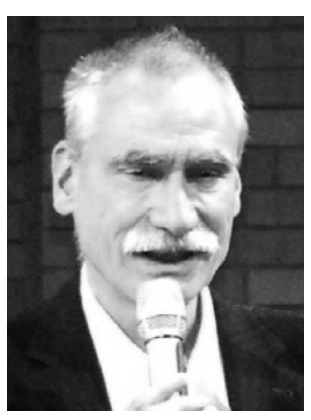

Wolfgang Nitze (59) studierte an der Staatlichen Ingenieurakademie Gauss Berlin Messund Regeltechnik und anschließend an der Evangelischen Fachhochschule für Sozialarbeit und Sozialpädagogik Berlin. Er leitete eine evangelische Kindertagesstätte und wechselte dann in die Berliner Verwaltung, wo er bei den Jugendämtern Neukölln und Kreuzberg tätig war. Seit 2001 ist er im Gesundheitsamt Friedrichshain-Kreuzberg von Berlin beschäftigt.

E-Mail w.g.p.nitze@arcor.de

ken, prozessorientiertes Handeln, erfahrungsorientiertes Lernen und qualitätsbewusstes Denken. Auf dieser Basis konnten trag- und zukunftsfähige Konzeptionen entwickelt werden, die in der Umsetzung allen Bürgerinnen und Bürgern zugute kamen. Diese Zusammenarbeit erlaubte es, dass Projekte im Interesse der Klientel neue konzeptionelle Wege der Hilfe ausprobieren konnten. Wir empfanden dies als einen positiv kreativen Prozess im Sinne einer professionellen Organisationsentwicklung bezogen auf ein psychosoziales Bezugssystem vergleichbar mit einer Stadt mit 260.000 Einwohnern.

Es stellt sich uns nun die Frage, ob dieses System der Zusammenarbeit und Entscheidungsfindung in der Folge 
von Strukturveränderungen, wie die Bildung der Jobcenter und die Einführung des Arbeitslosengeldes II bei einer gleichzeitigen Zunahme der Betonung von Sparzwängen an seine Grenzen gestoßen ist.

Bezüglich der Frage, wie Führungskräfte sozialer Organisationen ihre Situation einschätzen und welche persönlichen Eigenschaften sie für ihre Arbeit für besonders wichtig halten, haben die Beiträge aus dem Buch »Mutmacher « verdeutlicht, dass die fachpolitische und gesellschaftliche Komponente nicht außer Acht gelassen werden darf. Fachpolitisches Engagement und das Einwirken auf fachpolitische Entscheidungsprozesse sollten zum Repertoire einer Führungskraft gehören. Diese Fähigkeiten scheinen dringend notwendig, um in Zeiten der zunehmenden Liberalisierung, Globalisierung und der »Sucht nach mehr Qualität« einen Blick für das richtige Maß zwischen Effektivität, Effizienz und Ethik in der psychosozialen Arbeit zu behalten. Fachpolitische und gesellschaftliche Attribute können inzwischen nach unserer Erfahrung deutlich schwerer in den Arbeitsalltag von Führungskräften integriert werden.

Führungskräfte und Mitarbeiter werden zunehmend genötigt, immer mehr auf die Zahlen und immer weniger auf die tatsächlichen Inhalte zu sehen. Dieser einengende Prozess ist vergleichbar mit einer Einbahnstraße, in der ein Umkehren nicht mehr gestattet wird. Für Führungskräfte und deren Führungsverhalten bedeutet dieser Weg, notwendige inhaltliche und fachliche Freiräume weniger nutzen zu können. In der Folge müssen Führungskräfte auch im psychosozialen Bereich immer mehr als Manager fungieren. Das meint hier aber fast ausschließlich den Blick auf Kostenersparnis bei noch annehmbarer Qualität, und soll hohe Qualität und umfangreiche Angebote als vermeintlichen Luxus ausschließen. Soziale Dienstleister müssten sich dieser Entwicklung beugen und anpassen, um ihre Existenz nicht zu gefährden und damit wird die Gefahr größer, ausschließlich in den Kategorien Effektivität und Effizienz zu denken. Die Konsequenz wäre, dass fachliche Inhalte auf der Strecke bleiben müssen.

Wir wollen nicht missverstanden werden: Auch Führungskräfte in sozialen Einrichtungen müssen wirtschaftlich denken und handeln. Allerdings müssen diese notwendigen Handlungsmaximen sich im Gleichgewicht mit einer offensiven Diskussion fachlicher Art halten, um die notwendige Qualität von psychosozialen Angeboten zu garantieren.

Unsere Erfahrungen sind, dass in Zeiten der zunehmenden fiskalischen Misere das Formale zunehmend gegenüber dem Fachlichen in den Vordergrund drängt. Der Verwaltungsakt wird damit zu einer Größe erhoben, die ihm nicht zugestanden werden darf, da er überhaupt nur zur Erfüllung von inhaltlichen Aufgaben existiert und kein Selbstzweck ist. Führungskräfte stehen dabei heute und in der Zukunft vor einer anspruchsvollen Herausforderung.

Partnerschaftliche Organisationsentwicklung hat in den psychosozialen Bereich einschließlich des öffentlichen Dienstes zu wenig Eingang gefunden und wird kaum als
Lenkungsaufgabe verstanden. Zeitdruck, Arbeitsverdichtung, negative Konkurrenz, Orientierung an kurzfristigen Erfolgen, Machterhalt, unkritische Sparvorgaben, Kompromisslosigkeit in Fachfragen, das angespannte Verhältnis von Führungskräften zu ihren Mitarbeiterinnen und Mitarbeitern, das Abhängigkeitsverhältnis von Geldgebern zu Geldnehmern, die Forderung nach Unterwerfung von sozialen Unternehmen unter die Regeln des freien Marktes - all das spricht gegen zukunftsfähige Lösungen.

\section{»Die >Sucht nach mehr Qualität verstellt Effektivitat, Effizienz und Ethik Effektivität, Effizienz und Ethik«}

Kurzfristige Problemlösungen im Kleinen haben meist deutliche Auswirkungen auf einen anderen Bereich im sozialen und gesellschaftlichen System. Für umfassende Lösungsansätze muss Führung neu gedacht werden. Dazu gehört, dass Partnerschaft auch als Partnerschaft - also das Handeln auf Augenhöhe - verstanden wird. Dazu gehören soziale Tugenden wie Beziehungs-, Kommunikations- und Vertrauensfähigkeit. Unerlässlich ist dabei der Wille, Projekte und Vorgänge positiv abzuschließen. Das heißt: Führungskräfte müssen in Prozessen denken und sie müssen in ihren Entscheidungen die Auswirkungen auf andere Bereiche im Blick haben.

Führungskräfte sollten in der Lage sein, Entwicklungen von unten aufzunehmen und mit den Mitarbeiterinnen und Mitarbeitern gemeinsam inhaltlich zu gestalten, also Erfahrungen aus Praxis bei der Entscheidungsfindung zu berücksichtigen. Führungskräfte in sozialen Dienstleistungsunternehmen könnten dabei auf Leitgedanken zurückgreifen, um einer angemessenen Verbindung von fiskalischen und fachlichen Ansprüchen zu realisieren:

- Soziale Kompetenz führt zu disziplinierter Selbstreflektion.

- Disziplinierte Selbstreflektion führt zu Transparenz.

- Transparenz macht Beziehungsfähigkeit möglich.

- Beziehungsfähigkeit erhöht Veränderungsbereitschaft.

- Veränderungsbereitschaft beinhaltet, neue Ideen zu verwirklichen.

- Neue Ideen zu verwirklichen heißt, das Interesse von Klienten und Mitarbeitenden besser wahrzunehmen.

Fachlichkeit und Blick auf Effizienz müssen in einer Führungsposition bei sozialen Dienstleistungserbringern ihren gleichberechtigten Anteil haben und die Führungskräfte sollten den Mut haben, trotz ökonomischer Zwänge beide Anteile im Führungsalltag zu leben. Sie haben unserer Ansicht nach die Verpflichtung, sich in ihrer Position und unter Berücksichtigung ihrer Möglichkeiten darum zu bemühen, die gesellschaftliche Entwicklung positiv zu beeinflussen und zu gestalten. 
Die Beiträge des Buches »Mutmacher « zeigen, dass es verschiedene Möglichkeiten gibt, sich mit einer neuen Situation auseinanderzusetzen und Lösungen zu finden. Im Ergebnis zeigt sich, dass es von Bedeutung ist, die Zeichen der Zeit zu erkennen, die Erfahrungen aus Wissenschaft und Praxis zeitnah zu berücksichtigen und ohne Angst neue Wege zu beschreiten, die trotz ihrer Unterschiedlichkeit ein gemeinsames Ziel haben können: die fachlich fundierte, angemessene und dennoch effiziente Versorgung von hilfebedürftigen Menschen.

\section{Literatur}

Fischer, Roger; Ury, William; Patton, Bruce (2001): Das Havard-Konzept. Campus Verlag, Frankfurt am Main/New York.

Löhner, Michael (2005): Führung neu denken, Das Drei-Stufen-Konzept für erfolgreiche Manager und Unternehmen, Campus Verlag Frankfurt am Main/New York.

Knorr, Friedhelm; Halfar, Bernd (2000): Qualitätsmanagement in der Sozialarbeit. Walhalla Fachverlag, Regensburg.

Kießling, Annette (jetzt Börner); Meese, Jörg (2006): Qualitätsmanagement nach DIN EN ISO 9001:2000 in pädagogisch orientierten Dienstleistungsbetrieben; Tectum Verlag, Marburg.

Meese, Jörg; Nitze, Wolfgang Hg. (2007): Mutmacher, Ansichten zur Lage im Psychosozialen System. Ausgesuchte Beiträge, Tectum Verlag, Marburg.

Rudat, Volker (1997): Die Grundlagen einer PraxisTheorie der Organisationsentwicklung. Universität Bremen: ABO-Projekt »Wandel in Organisationen «. Thema des Projektteams: »Wandel der Universität Bremen «, Bremen.

Tretter F. (1998): Ökologie der Sucht, Hogrefe Verlag, Göttigen.
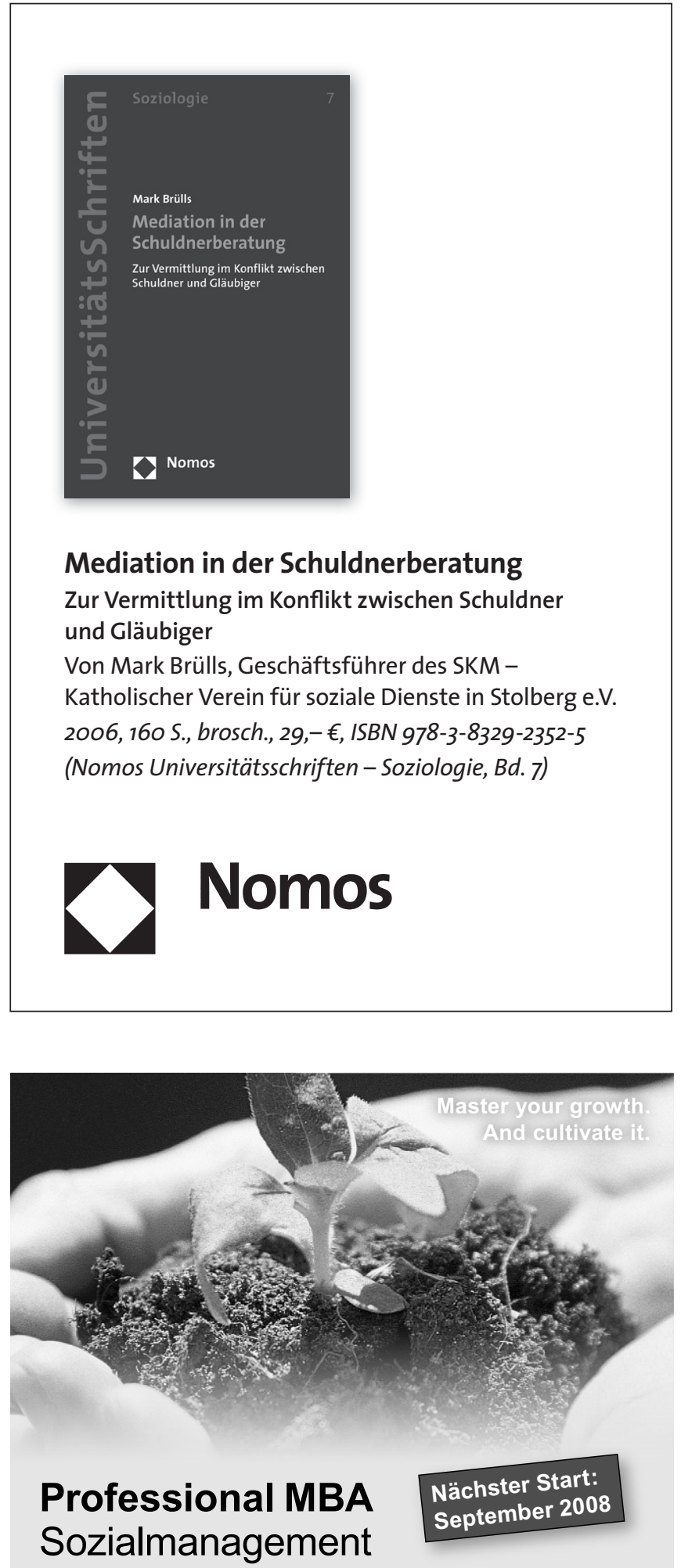

Zielgruppe: Aktive und zukünftige Führungskräfte in Sozialund Nonprofit Organisationen

Dauer: - 24 Monate: Professional MBA Sozialmanagement

- 18 Monate: Universitätslehrgang Sozialmanagement

- Berufsbegleitende Blockveranstaltungen

Preis: - 24 Monate: EUR 14.000,-

- 18 Monate: EUR 9.100,-

Kontakt: Renate.Braun@wu-wien.ac.at +431313365820 www.executiveacademy.at 